\title{
An Efficient Contact Tank Design for Potable Water Treatment
}

\author{
Ender DEMIREL ${ }^{1}$ \\ Mustafa M. ARAL ${ }^{2}$
}

\begin{abstract}
In this study, a second order accurate Large Eddy Simulation (LES) model is used to simulate three-dimensional turbulent flow and disinfectant transport in a contact tank. The hydraulic and mixing indexes of the tank are evaluated based on computational tracer studies. It is shown that the energy consumption rates of the contact tank can be reduced by the use of the proposed slot-baffle design instead of the conventional baffle system that is used in these facilities. The proposed slot-baffle design improves the hydraulic efficiency by $44 \%$, mixing efficiency by $42 \%$ and reduces the energy required to drive the flow through the system by $43 \%$. The outcome reported in this study shows that the energy consumption in water treatment plants can be significantly reduced when the novel baffle design is implemented on existing contact tanks without expensive infrastructure modifications.
\end{abstract}

Keywords: Contact tank, treatment of potable water, CFD, turbulence, LES.

\section{INTRODUCTION}

Contact tanks are critical infrastructures that are used in water treatment facilities that treat potable water with chlorine or ozone. Historically, as a design criteria, the design of these facilities are not based on minimum energy consumption during the operation of the contact tank. The contact tank design is based on plug flow assumptions where viscous effects are not considered and more emphasis is placed on sufficient treatment. This design approach may over or under estimate the mixing condition and the disinfectant use without much consideration given to energy consumption during treatment. In large population centers where high volumes of water are treated and consumed daily, high energy consumption rates have now become a critical impediment for water treatment at these facilities. In modern cities and municipalities, parallel to the population growth, the energy intensive treatment methods such as ozone treatment is also becoming more popular. Accordingly, improvement of hydraulic and mixing efficiency of contact tanks that would also reduce the energy demand is now in the forefront of academic research and applications. An efficient contact system

Note:

- This paper has been received on June 23, 2017 and accepted for publication by the Editorial Board on January 30, 2018.

- Discussions on this paper will be accepted by May 31, 2018.

- DOI: $10.18400 /$ tekderg.322491

1 Eskisehir Osmangazi Uni., Department of Civil Engineering, Eskişehir, Turkey - edemirel@ogu.edu.tr

2 Bartın University, Department of Civil Engineering, Bartın, Turkey - mustafaaral@bartin.edu.tr 
design that addresses effective water treatment while using less energy is now important and is the topic of this study.

In most Turkish cities, potable water is treated with chlorine. Ozone treatment is an energy intensive treatment process which is mostly used in developed countries with a gradual trend of moving to ozone treatment in developing countries as well. Ozone treatment is a more effective water treatment process and an eco-friendly application, but it is an expensive process. Thus, an efficient contact tank system is more important for ozone treatment since ozone cannot be dissolved in water as much and as quickly as cholorine. In a classical contact tank design, baffles are placed at specific locations inside the tank to increase the contact time of water and the disinfectant. In Turkey contact tanks are designed based on the assumption of plug-flow conditions which maintain the contact time for about 20 minutes [1]. However, recirculating dead zones that form in the vicinity of the corners of baffles due to the viscous and turbulent effects and the formation of high velocity jet zones in the remaining area of the mixing chamber cause the injected concentration of the disinfectant to leave the contact tank without reaching the effective disinfection contact time. This undesirable condition is identified as short-circuiting. Short-circuiting effects reduce the hydraulic and mixing efficiency of the contact tank and may yield high residual chlorine concentrations in water at the outlet. This is a risky condition for both human health and also for the environment. The short-circuiting effects also increases the energy consumption rates in water treatment plants where energy intensive treatment methods such as ozone and ultraviolet are used. It is important to remember that almost $90 \%$ of the total energy consumption in an ozone treatment plant is used for ozone generation [2]. Thus, significant importance is given to research and development studies where specific designs are considered to reduce energy consumption in ozone treatment through improved mixing more than the chlorine treatment facilities.

Hydraulic and mixing efficiency of contact tanks can be evaluated using the indexes that can be obtained from the Residence Time Distribution (RTD) and cumulative RTD plots. These distributions are obtained from tracer studies, where the injected concentration of the disinfectant at the inlet is monitored at the outlet of the tank to obtain RTD and the cumulative RTD plots. The cumulative RTD plot is the integral of the RTD plot with respect to time. Tracer studies can be conducted experimentally by monitoring the concentration of a tracer such as the fluorescent dye (Rhodamine B) by using fluorometer at the outlet [3, 4]. More recently, developments in computational methods made it possible to design contact tanks using Computational Fluid Dynamics (CFD) methods more economically than the conventional experimental methods $[5,6,7,8]$ where a tracer study can be conducted numerically. In these studies the motion of the injected tracer with the turbulent flow is numerically simulated to obtain the RTD and cumulative RTD plots which show the variation of concentration and cumulative concentration of the tracer at the outlet, respectively $[7,8]$. Finally, hydraulic and mixing efficiency of the contact tank is evaluated using the indexes which are obtained from the RTD and cumulative RTD plots.

Turbulence model employed in numerical simulations have significant effect on the results obtained. To reduce computation time, RANS based turbulence models such as $k-\varepsilon, k-\omega$ and low Reynolds number models [4] have been extensively used in the design of large scale treatment plants. Recently, LES models in which the unsteady variation of large turbulent fluctuations can be evaluated, have gained significant attention with the development of 
computer technologies that reduce computational time [6,9]. It has been reported in the literature that the recirculation zones that form in the vicinity of the corners of the baffles could not be detected when the RANS based turbulence models are used, while those recirculation zones could be simulated with LES on a fine resolution computational mesh. Moreover, turbulence induced mixing of the disinfectant with the flow could only be simulated with LES models [6, 9], which is critical for the present problem.

In this study, turbulent flow in the contact tank with four chambers is simulated using a second order accurate computational model in space and time. LES turbulence model is employed in order to calculate the unsteady variation of turbulent eddies near the corners of the baffles. Mixing of the disinfectant with the the turbulent flow is simulated by solving the Advection-Diffusion equation with the momentum equations simultaneously. Accuracy of the present numerical model is evaluated based on the comparison of the numerical results with the previously reported results in the literature. It is shown that the hydraulic and mixing efficiencies of the contact tank can be increased with the proposed slot-baffle design and energy consumptions in water treatment plants can be reduced by implementing the proposed design in existing water treatment plants without costly infrastructure modification.

\section{PROBLEM DEFINITION}

Schematic view of the flow in the contact tank is shown in Figure 1. Water enters to the tank with constant flow rate $Q=0.2 \mathrm{lt} / \mathrm{s}$ and the depth of the flow is maintained at $h=0.21 \mathrm{~m}$ when the mass conservation is achived inside the domain after a certain time. Height of the baffles are selected as $0.18 \mathrm{~m}$ and the horizontal spacing between the baffles is $w=0.113 \mathrm{~m}$. Flow properties and dimensions of the tank are selected to be the same as previous studies

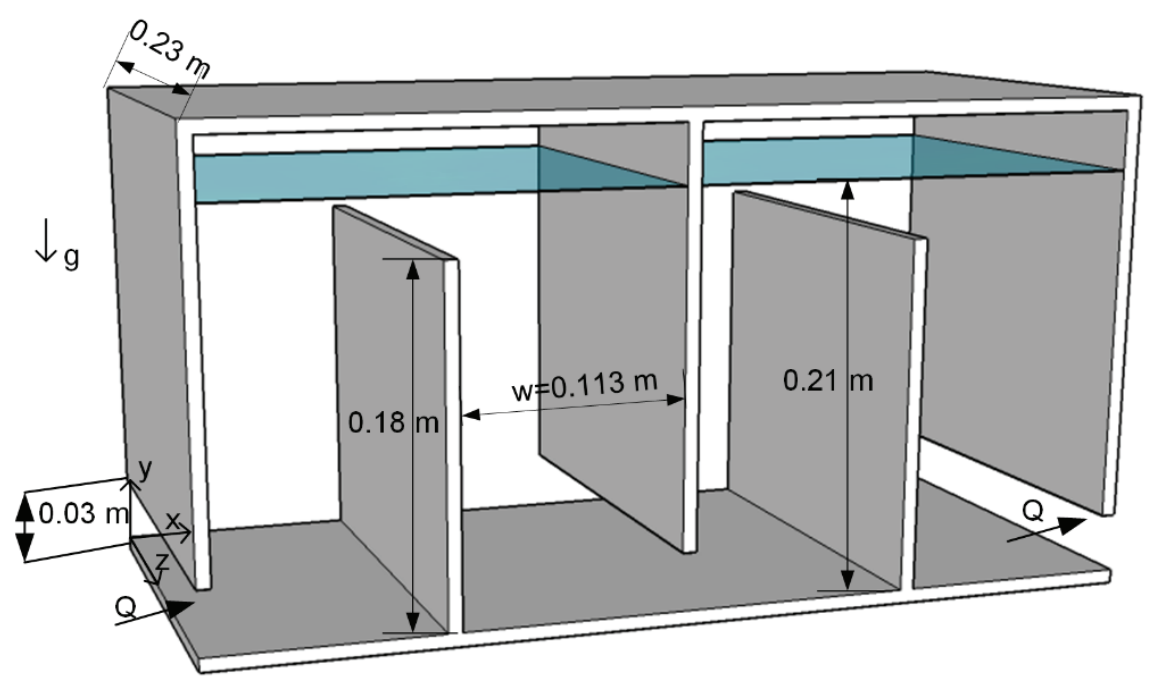

Figure 1. Three-dimensional schematic view of the flow inside the contact tank 
in order to compare the numerical results with the previously reported results $[6,10]$. In this study, a truncated version of the contact tank is used applying periodic boundary conditions at the inlet and outlet even though experimental studies were conducted using the same tank having 12 chambers. Prescribing inlet boundary condition for turbulence at the inlet requires a cumbersome process for the simulation of turbulent flows. Boundary conditions for the turbulence quantities at the inlet do not need to be prescribed in this study since periodic boundary conditions are used for all flow variables at the inlet and outlet of the computational domain.

The Reynolds number of the flow is calculated based on the average velocity at the inlet and the equivalent hydraulic radius as $R e=U_{a v} R / \vartheta=2740$. Here $R=\sqrt{4 A / \pi}$ and $A$ is the area of the cross-section at the inlet. No-slip boundary conditions are imposed at solid walls and symmetry boundary condition is used at the free-surface based on the assumption of no-shear since the surface waves can be neglected $[2,6]$.

\section{COMPUTATIONAL MODEL AND VALIDATION}

Reyndols-averaged Navier-Stokes (RANS) models such as $k-\varepsilon, k-\omega$ and low Reynolds closure models have been extensively used in the literature for the simulation of the turbulent flow in the contact tank. However, recent studies in the literature have postulated that the time-varying turbulent eddies in the vicinity of the baffle corners affect not only the mean flow structure inside the tank but also the mixing of the disinfectant with the flow $[6,9]$. Time variation of turbulent eddies cannot be simulated accurately using RANS based turbulence models due to the fact that only time-averaged values of the flow are considered in RANS applications. Thus, in this study numerical simulation of the turbulent flow inside the contact tank is performed employing LES turbulence model, which is the main difference from the previously published study of the authors [17]. Governing equations for the LES will not be repeated here since conventional equations of LES can be found in the literature $[6,9]$.

In the numerical simulation of fluid flow using LES, large eddies are resolved directly by the computational mesh and the small eddies are modeled using a sub-grid scale (SGS) model. Thus, the resolution of the computational mesh should be fine enough to capture the turbulence structure. The most classical SGS model is Smagorinsky SGS model in which the $C_{s}$ Smagorinsky coefficiency is kept constant at 0.1 . Some drawbacks may occur when this coefficient is kept constant in the computational domain throughout the numerical simulation. This coefficient may take smaller values near the solid walls depending on the flow properties. In addition, $C_{s}$ coeficient should take negative values to calculate the backscattering effects due to energy transfer from small to large scales. Fixing the Smagorinsky coefficient at a specific value may reduce the accuracy of the computational model. In this study, Dynamic Smagorinsky LES [11] approach is employed in which the Smagorinksy coefficienct is calculated dynamically from the interior solution of the domain at every time step of simulation in order to exclude the exsessive dissipation near the walls and to consider the energy backscattering effects. 


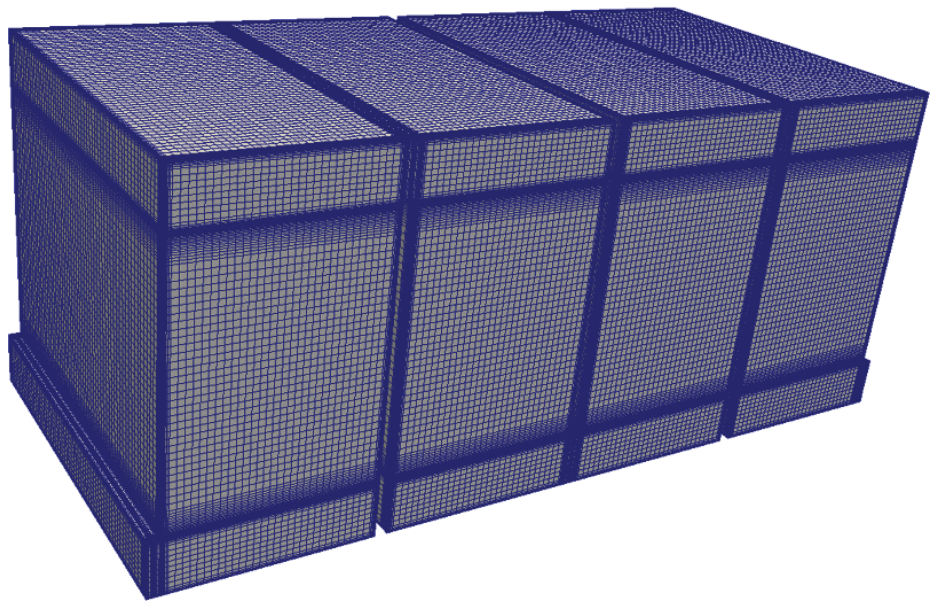

Figure 2. Three-dimensional view of the structural mesh

An open source CFD code OpenFOAM is used in numerical simulations conducted in this study [12]. In the numerical solution, convective and diffusive terms in the governing equations are discretized using second order accurate LinearUpwind and linear methods, respectively, unsteady terms are discretized using second order accurate backward method. Thus the numerical model employed in this study is second order accurate in space and time to reduce the truncation errors that arise from the discretization of the partial differential equations. A structured grid system is used in the numerical simulations clustering the computational mesh near the walls and free-surface where temporal and spatial variations of flow variables are to be significant. About 1.8 million computational cells are used in the computational mesh shown in Figure 2 ensuring that the dimensionless wall distance adjacent to the solid wall is $y^{+}<3$. Thus, the viscous sub-layer is resolved in the LES accurately without using wall functions, which may reduce the accuracy of the LES solutions near the walls.

Time step size $\Delta t$ in the unsteady solution of the governing equations is selected according to the Courant-Friedrichs-Lewy $(C F L)$ stability condition such that $C F L=0.5$ during the numerical simulation. High-resolution numerical solutions in space and time are performed in parallel on the resources of TRUBA (Turkish Academic Network and Information Center) with 112 computational nodes within the OpenFOAM on the domain decomposition approach. In parallel computing, computational domain is decomposed into the same number of available nodes calculating each sub-domain on different processor and enabling messaging of the processors with each other during the simulation. At the end of the parallel computing, decomposed solution domains are reconstructed to obtain the solution in the overall domain. Implementing parallel computing strategy allowed us to simulate the problem with high-resolution numerical solution at acceptable computational times. 


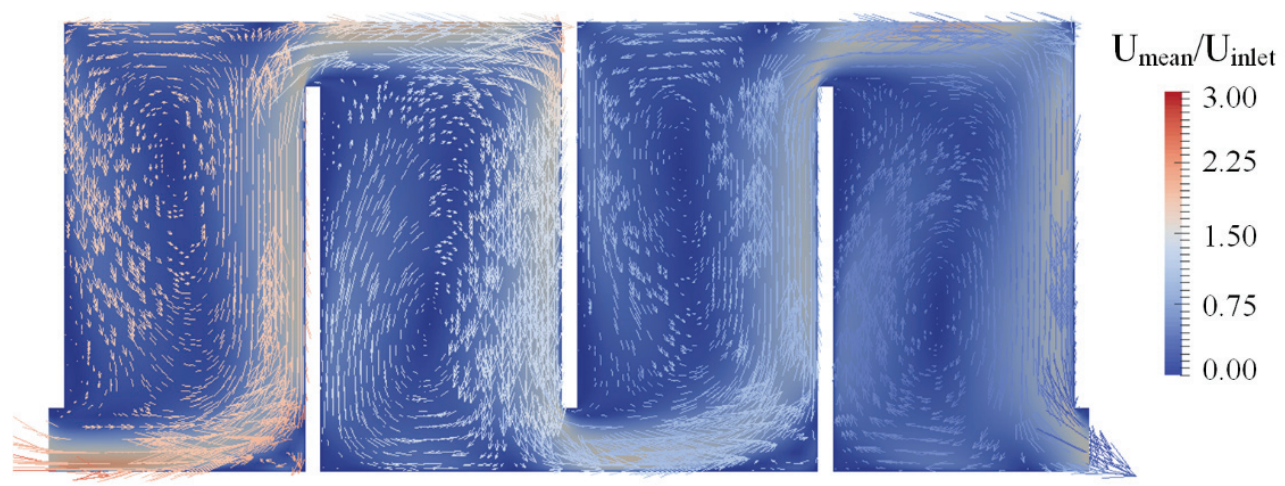

(a)

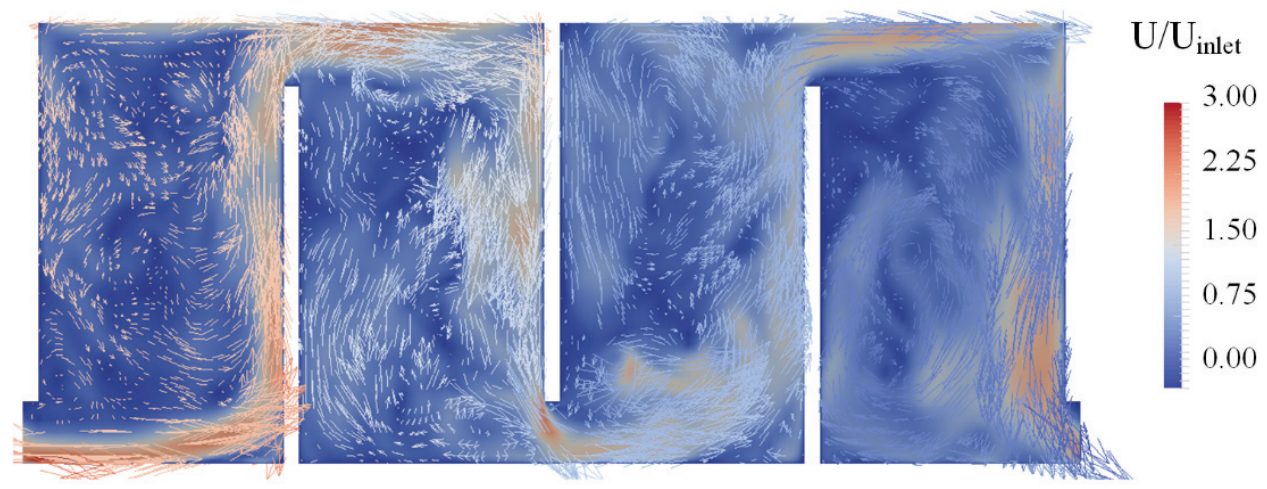

(b)

Figure 3. Velocity magnitudes and vectors at the vertical plane passing at the center of the tank $(z=b / 2)$; (a) Time-averaged velocity; (b) Instantenous velocity

Instantenous flow properties are used to calculate the time-averaged value of a variable during the simulation of turbulent flows using LES. It is required to simulate the flow long enough to obtain time-averaged flow field excluding the severe variations in flow properties that occur during the initial stage of the simulation [13]. In order to exclude the effects that arise from the initial conditions, time-averaging was performed starting from $t=200 \mathrm{~s}$ to 600 s. Velocity magnitudes and vectors at the vertical plane passing at the center of the tank are shown in Figure 3 for both time-averaged and instantenous flow fields. As seen in Figure 3, large recirculation zones occur due to wall effects near the baffles and this also forms a jet zone with increased velocity especially at the inlet of the chambers extending towards the opposite wall. Magnitude of the flow velocity in the jet zone is decreasing along the flow direction due to viscous and turbulence effects. Turbulence fluctuations near the baffles form small recirculation zone at the inlet of each chamber. Demirel and Aral [13] showed that the recirculation and jet zones could be separated using the vorticity field and the definition of Lamb vector to define a volumetric efficiency coefficient for the evaluation of hydraulic efficiency of the contact tank. Short-circuiting in the tank causes the injected concentration 
of disinfectant at the inlet to advect with the high velocity parcels and to leave the contact tank without effective disinfection. This reduces the overall efficiency of the contact tank. Viscous and turbulence induced short-circuiting effects connot be predicted for the contact tanks where the design is based on the plug-flow assumption. Thus, high-resolution numerical models should be employed for the design of contact tanks that include viscous and turbulence effects. As will be discussed at the latter part of the study, proposed slot-baffle design increases both hydraulic and mixing efficiency of the contact tank while reducing the momentum of the jet zones.

As seen in Figure $3 b$, unsteady effects in turbulence field significantly alter the flow field. LES turbulence models should be used in order to simulate large eddies in the tank accurately. It is also critical to use LES model in order to simulate the advective diffusion of the tracer with the turbulent flow. Numerical simulations in which the RANS based turbulence models are used could not detect the recirculation zones near the inlet of the chambers and the accuracy of the simulation of the tracer with the turbulent flow may reduce as well. Thus, it is suggested to use computational models that can capture the large eddies in the flow field for proper advective dissusion analysis.

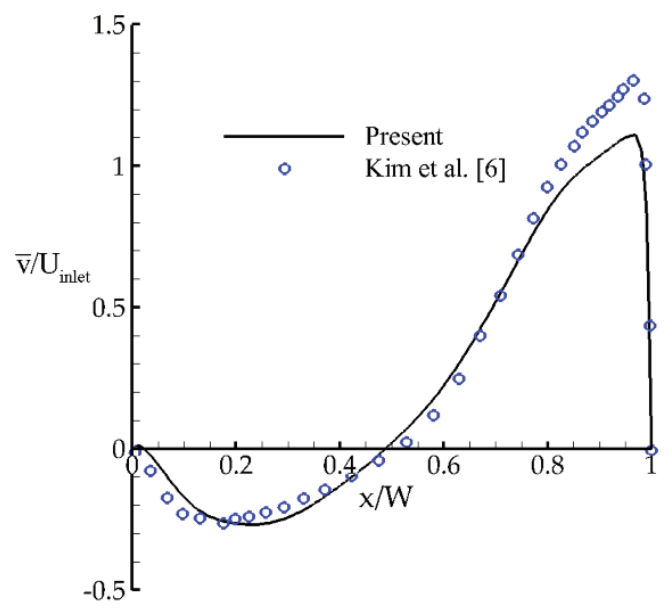

Figure 4. Variation of the time-averaged vertical velocity $(\bar{v})$ along the chamber width $(W)$

Variation of the time-averaged vertical velocity is compared with the numerical results in the literature at the elevation $y / H=0.27$ in the third chamber in Figure 4. Negative vertical velocities between the baffle and the center of the chamber indicate a large recirculation zone inside the chamber. The present computational model could detect this recirculation zone accurately. The magnitude of the velocity in the jet zone is underestimated in comparison to the previous numerical results. This could be due to time averaging that was not performed for a long time in the literature since the flow velocities are high at the initial stage of the simulation and magnitude of the velocity in the jet zone is reduced during the simulation. 
Thus, the magnitude of the velocity is reduced in the jet zone when time averaging is performed for a long time, as previously reported in [13].

\section{TRANSPORT MODEL AND VALIDATION}

The transport of concentration of disinfectant injected at the inlet is traced using the following advection-diffusion equation:

$$
\frac{\partial \bar{C}}{\partial t}+\bar{u}_{j} \frac{\partial \bar{C}}{\partial x_{j}}=\frac{\partial}{\partial x_{j}}\left(\left(D+D_{t}\right) \frac{\partial \bar{C}}{\partial x_{j}}\right)
$$

Here $u_{j}$ is the velocity component along the $j$-direction $(x, y$ and $z), t$ is time, $x_{i}$ and $x_{j}$ are Cartesian coordinates, $\bar{C}$ is filtered concentration, $D$ is molecular diffusion, $D_{t}$ is turbulent diffusion, which can be calculated as $D_{t}=v_{t} / S c$. Here $v_{t}$ is turbulent viscosity which is calculated during simulation and $S c$ is the Schmidt number which is set to 1000 in order to be consistent with the numerical studies conducted in the literature $[6,9]$.

"Frozen flow" approach has been extensively used in the tracer studies conducted in the literature. In this approach, steady state flow field is obtained first, then Equation (1) is solved using the steady state flow field. In this approach, interaction of the disinfectant and the turbulent flow cannot be simulated realistically since advection-diffusion equation and flow equations are not solved simultaneously. In the present study, the aim is to simulate the turbulence induced mixing of the disinfectant by solving the advection-diffusion equation with the flow equations simultaneously. In order to achieve this, standard solver pimpleFoam that is available in OpenFOAM is modified to include the solution of the advection-diffusion equation with the flow equations for a new variable of concentration $\bar{C}$. Employing an open source computer code in the present study allows us to modify the standard solver depending on the properties of the problem.

In order to validate the solution of the tracer study, tracer is injected at the inlet of the tank shown in Figure 1 during $2.5 s$ and variation of the concentration at the outlet is monitored during the numerical simulation. Concentration of the tracer is non-dimensionalized in the following form in order to be consistent with the previous studies reported in the literature and to satisfy the volume conservation of the injected concentration $[2,6,9]$ :

$$
E(\theta)=\frac{\bar{C}}{\frac{\bar{C}_{\text {initial }} T_{\text {injection }}}{\tau}}
$$

Here $T_{\text {injection }}$ is the duration of injection $(2.5 s), \bar{C}_{\text {initial }}$ is the tracer concentration at the inlet ( $\left.\bar{C}_{\text {initial }}=1\right), \tau$ is the theoretical residence time. Theoretical residence time is defined for the plug-flow as $\tau=\forall / Q[2,6]$. Here $\forall$ is the volume of the tank $\left(m^{3}\right)$ and $Q$ is the flow rate in the tank $\left(\mathrm{m}^{3} / \mathrm{s}\right)$. Theoretical residence time is calculated as $109.2 \mathrm{~s}$ for the present problem. 
RTD and cumulative RTD plots are compared with the results reported in the literature in Figure 5a and Figure 5b, respectively. Time axis is non-dimensionalized with respect to the theoretical residence time as $\theta=t / \tau$.

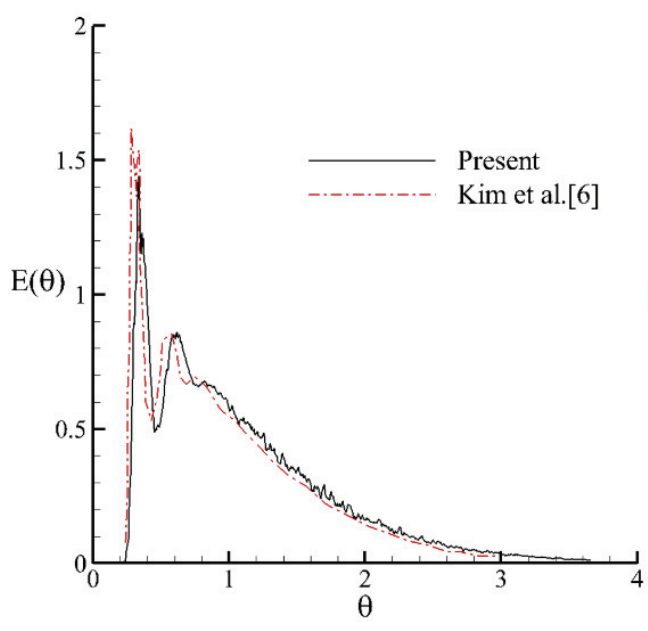

(a)

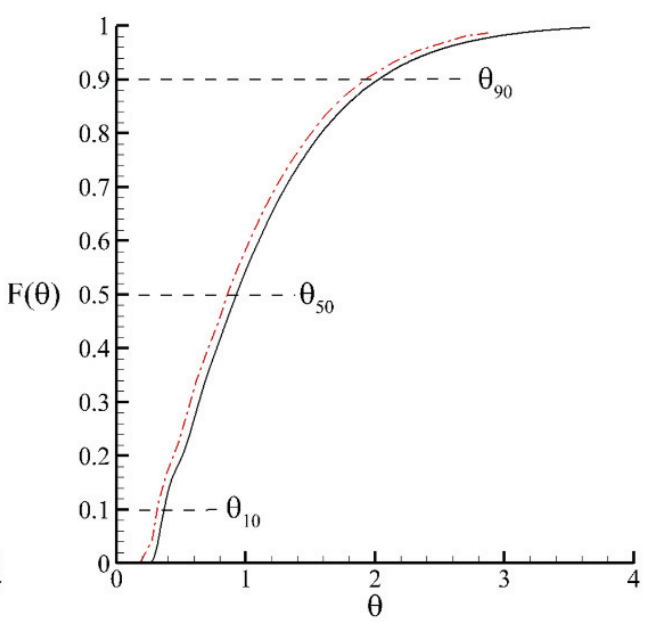

(b)

Figure 5. Comparison of the dimensionless concentration of disinfectant at the outlet with the literature: (a) RTD plot; (b) cumulative RTD plot

The first peak in Figure 5a indicates the tracer concentration that is transported by the high velocity parcels in the jet zone and leaves the contact tank after a short time without effective disinfection. Some part of the injected concentration is transported by the jet zone and the remaining part of the concentration is trapped in the reciurculation zones as shown in Figure 3 for a time duration. The trapped volume of the concentration emerges from the recirculation zones and leaves the contact tank at a later time, which is indicated by the second peak observed in Figure 5a. The time value at which the tracer concentration first observed at the outlet indicates that the short-circuiting effects are significant. Short-circuiting reduces the efficiency of the contact tank and causes more disinfectant to be used for effective disinfection and this increases the energy consumption rates during treatment. As seen in Figure $5 \mathrm{~b}$, total injected concentration leaves the contact tank at about $\theta=3.66$. Tracer results are in good agreement with the previous results showing that the present computational model could accurately calculate the turbulence induced mixing in the tank. The small differences observed may be associated with the resolution of the computational mesh since the RTD and cumulative RTD plots shift to the left when the resolution of the mesh reduces. It should be noted that the present mesh is finer than the mesh used in the literature [6].

Indexes are obtained from the cumulative RTD plot to evaluate the hydraulic and mixing efficiencies of the contact tank. The most common index used for the hydraulic efficiency of 
the contact tank is $\theta_{10}$, which can be defined as the time required for $10 \%$ of the injected tracer to pass at the outlet. This index is also called the "baffle factor." According to the EPA [14] regulations, a contact tank is classified as "poor" for $\theta_{10}<0.3$, "compromising" for $0.3<\theta_{10}<0.5$, "good" for $0.5<\theta_{10}<0.7$ and "excellent" for $0.7<\theta_{10}<1.0$. The baffle factor becomes unity for ideal plug-flow conditions. The present contact tank is classified as compromising since the baffle factor is determined to be 0.369 from Figure $5 \mathrm{~b}$. Another type of efficiency index for a contact system is the "mixing efficiency," which is associated with the degree of the mixing of disinfectant with the water. The most common index for the evaluation of the mixing efficiency is the Morrill (Mo) index [14]. The Mo index is defined as the ratio of the time to pass the $90 \%$ of the injected concentration to the time to pass $10 \%$ of the injected concentration $\left(M o=\theta_{90} / \theta_{10}\right)$. The Mo index is unity for the ideal plug-flow and it takes the maximum value 21.9 [14]. EPA regulations suggest that $M o \approx 2$ for an ideal contact tank. Dispersion index is the other index for the evaluation of mixing efficiency of contact tank, which is defined as [15]:

$\bar{\sigma}=\frac{\sigma_{\theta}^{2}}{\theta_{m}^{2}}$

Here $\sigma_{\theta}^{2}$ is the variance of the RTD plot and $\theta_{m}$ is the mean of the RTD plot, which are defined as:

$$
\begin{aligned}
& \sigma_{\theta}^{2}=\frac{\int_{0}^{\infty} \theta^{2} E(\theta) d \theta}{\int_{0}^{\infty} E(\theta) d \theta}-\theta_{m}^{2} \\
& \theta_{m}=\frac{\int_{0}^{\infty} \theta E(\theta) d \theta}{\int_{0}^{\infty} E(\theta) d \theta}
\end{aligned}
$$

Dispersion index is a statistical definition associated with the dispersion of the RTD data around the mean of the RTD plot. Dispersion index takes values between 0 and 1 and it approaches 0 when the mixing in the tank increases. The aferomentioned indexes are determined from Figure $5 \mathrm{~b}$ for the contact tank shown in Figure 1 and these indexes are listed in Table 1. A Matlab program is developed for the calculation of the dispersion index based on the discrete RTD data.

Table 1. Efficiency indexes of the contact tank.

\begin{tabular}{cccc}
\hline$\theta_{10}$ & $\theta_{90}$ & Mo & $\bar{\sigma}$ \\
\hline 0.369 & 2.027 & 5.493 & 0.369 \\
\hline
\end{tabular}


The baffle factor $\theta_{10}$ in Table 1 indicates that the short circuiting effects in the contact tank are severe and the hydraulic efficiency is also low. Mixing efficiency of the contact tank is low since the value of Mo index is not close to 2 . The design considered in this study should increase both hydraulic and mixing efficiency of the contact system. The energy requirement to drive the flow from the inlet to outlet can be estimated from the following equation [8]:

$P_{f}=\Delta p Q / e$

Here $\Delta p$ is the pressure drop between the inlet and outlet, $Q$ is the flow rate passing at the contact tank, and $e$ is the coefficient of mechanical efficiency of the pump, which can be taken as $50 \%$. The pressure drop is calculated for the present tank as $\Delta p=9.45 \mathrm{kPa}$. Previous studies in the literature reported that the energy consumed by the pumps is significant in water treatment plants [15]. Energy losses in a contact tank are associated with the friction effects between the baffles and the flow. Previous studies in the literature suggested the use of additional baffles or turning vanes near the corner of the chambers to dissipate the energy of the recirculation zones and to increase the efficiency of the contact system. The use of additional solid regions located inside the tank reduces the effective volume of the contact system and causes extra energy losses. The proposed design that will be discussed next not only increases the hydraulic and mixing efficiency of the contact system but also reduces the friction induced energy losses.

\section{SLOT-BAFFLE DESIGN}

Aral and Demirel [17] proposed a novel baffle design to increase the efficiency of the contact system and this design is patented [18]. Three-slotted configuration of baffle design is depicted in Figure 6. Geometrical properties of the slots such as location, width, height and the numbers significantly affect the flow structure and efficiency of the contact system. The most efficienct configuration is investigated based on the successive numerical simulations and given in Figure 6. The main difference between the present and previous study [17] is that LES is used for the accurate simulation of the penetrating of the jets to the adjacent chambers. As discussed at the previous part of the study, realistic simulation of mixing of the disinfectant with the turbulence could be peformed using LES since the flow equations and advection-diffusion equation are solved simultaneously.

Averaged and instantenous velocity vectors for the slot-baffle design are shown in Figure 7. The jets emerging from the slots to the adjacent chamber reduce the momentum of the main jet which is the source of the short-circuiting and those jets imping to the neighboring dead zones also increase the mixing in the chamber and turn the dead zones into active mixing zones. Thus, the proposed slot-baffle design not only decreases the short-circuiting but also increases mixing in the contact tank. The main idea of the slot-baffle design is to reduce the friction losses and to increase the mixing efficiency while increasing the surface area between the fluid-fluid interfaces instead of increasing fluid-solid layers. Turning vanes were used and additional horizontal baffles were located on the vertical baffles in order to 
An Efficient Contact Tank Design for Potable Water Treatment

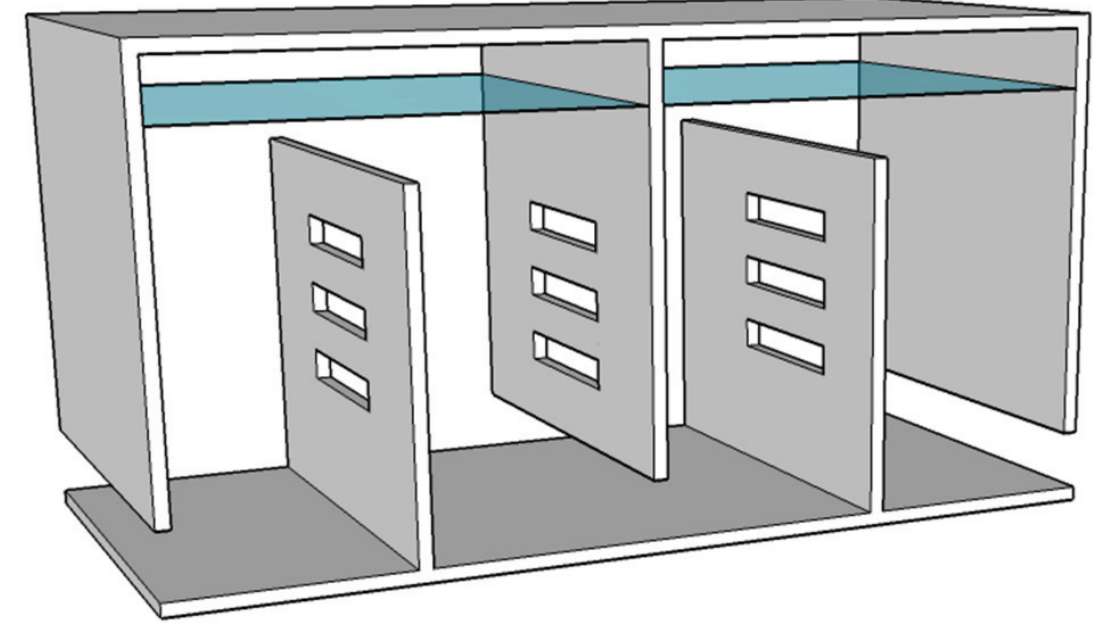

Figure 6. Implemantation of the slot-baffle design

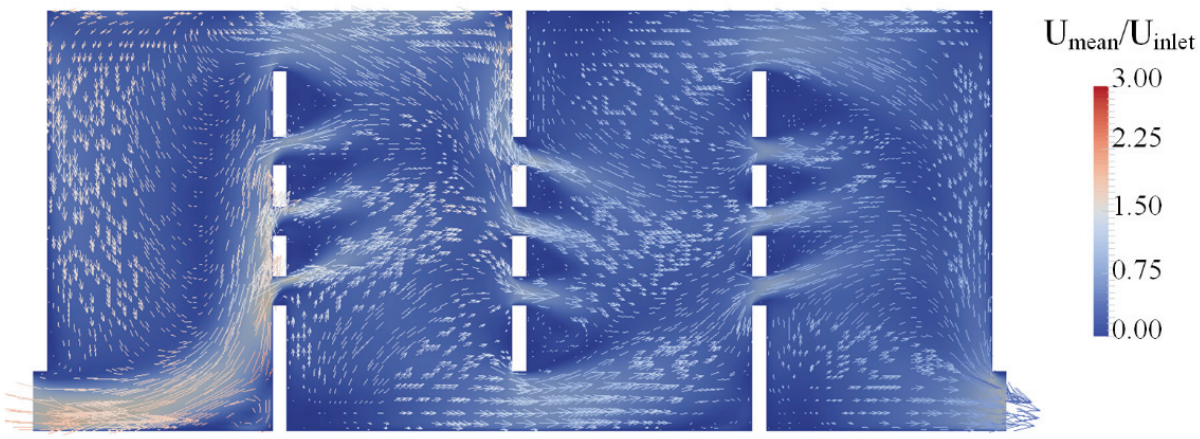

(a)

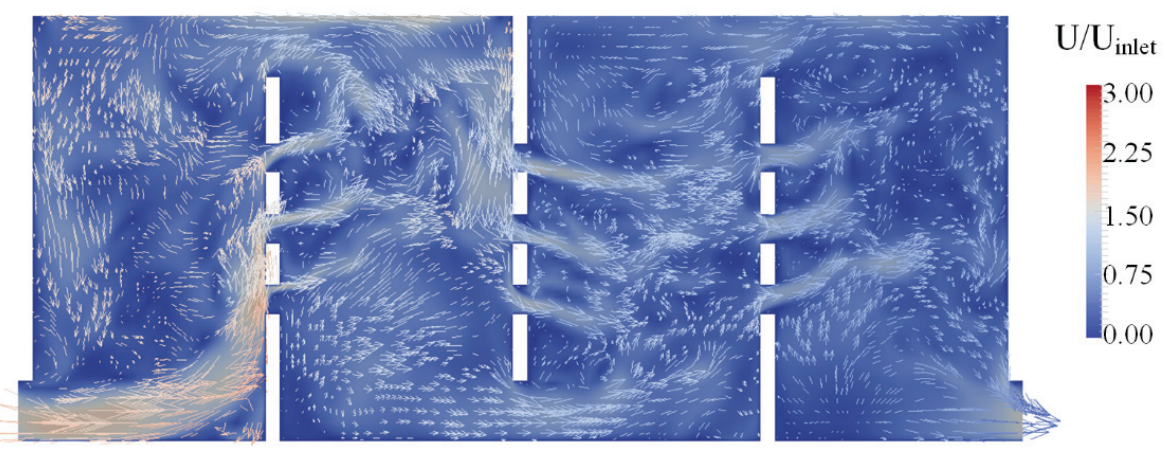

(b)

Figure 7. Flow field for the slot-baffle design; (a) Time-averaged flow field; (b) Instantenous flow field at $z=b / 2$ 
turn the low mixing zones to active mixing zones in the literature [8; 19]. However, those types of tank designs increases the energy requirements to drive the flow through the system since the energy losses increase due to the wall effects. The slot-baffle design reduces the energy losses that occur in the convetional baffle design. As seen in Figure 7b, the flow emerging from the slots significantly altered the instantenous flow field to increase the mixing phenomenon in the tank as well. This will be seen in the results of the tracer study.

Tracer study is performed for the slot-baffle design using the same methodology mentioned in the previous part and the results of the tracer study are given in Figure 8. The first peak in the RTD plot is reduced indicating that the hydraulic efficiency of the contact tank is increased since short-circuiting effects are significantly reduced. The second peak observed in the results of conventional baffle design is not seen in the slot-baffle design, which is another indication of turning low mixing zones into active zones. Shifting of the peak of the RTD plot to the right indicates increasing of the contact time in the tank.

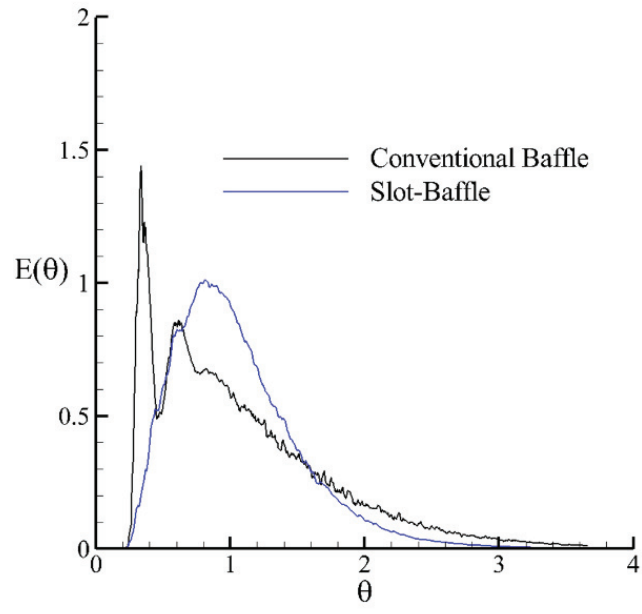

(a)

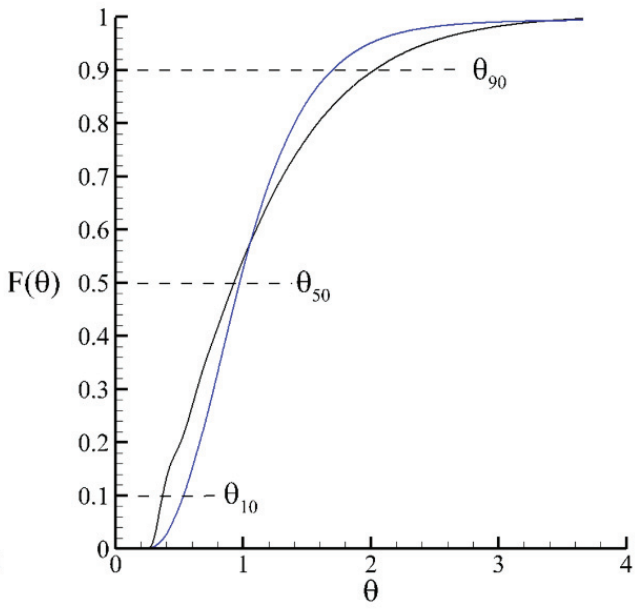

(b)

Figure 8. Comparison of tracer results of conventional and slot-baffle design; (a) RTD; (b) Cumulative RTD

As can be seen in the cumulative RTD plot in Figure 8b, the slot-baffle design altered the mixing indexes significantly. Efficiency indexes are determined from Figure $8 \mathrm{~b}$ for the slotbaffle design and compared with the convetional baffle design in Table 2. Hydraulic efficiency of the contact tank has increased about by $44 \%$ reducing the short-circuiting effects. Mixing efficiency of the contact tank has increased by $42 \%$ according to the Mo index and $46 \%$ according to the dispersion index. Increasing the hydraulic and mixing efficiencies will reduce the required dosage of chlorine to be used in the disinfection process and will also reduce the cost of disinfection as well as the health risk of the chlorine usage. This will also reduce the energy consumed in ozone treatment plants significantly since energy requirement is more pronounced when ozone is used. Comparison of the pressure drops at the last column of the table indicates that the slot-baffle design reduces the energy 
An Efficient Contact Tank Design for Potable Water Treatment

losses by $43 \%$. Thus, the energy requirements of the pump to drive the flow through the system will also be reduced.

Table 2. Efficieny indexes of conventional baffle and slot-baffle designs

\begin{tabular}{ccccc}
\hline Baffle design & $\theta_{10}$ & Mo & $\bar{\sigma}$ & $\Delta p / \rho\left(\mathrm{m}^{2} / \mathrm{s}^{2}\right)$ \\
\hline Conventional baffle & 0.369 & 5.493 & 0.369 & 9.45 \\
Slot-baffle & 0.532 & 3.192 & 0.201 & 5.41 \\
Improvement (\%) & 44.17 & 41.89 & 45.53 & 42.75 \\
\hline
\end{tabular}

\section{CONCLUSIONS}

Turbulent flow in a contact tank is simulated using a second order accurate computational model in this study. LES turbulence approach is employed in order to calculate the effect of time varying turbulent eddies on both flow field and the mixing of the disinfectant with water. A new computational model is developed to determine the efficiency indexes based on the tracer study. Numerical results are compared with the previous results in order to show the accuracy and reliability of the present numerical model.

Tracer studies show that the conventional tank designs suffer from high short-circuiting and low mixing. The proposed novel baffle design improves the hydraulic efficiency by $44 \%$ and mixing efficiency by $42 \%$. The proposed baffle design reduces high cholorine dosage used in traditional cholorine treatment plants, which may be risky for human and public health. Increasing hydraulic and mixing efficiency in the contact tank reduces the energy consumption rates in ozone treatment plants. Energy requirements of the pumps to drive the flow through the system are also reduced by the proposed baffle design since energy losses have been reduced by $43 \%$.

Short-circuiting effects cannot be predicted in the contact tanks when plug-flow assumption is used for the design. Viscous and turbulence effects significanlty alter the flow field inside the tank reducing the efficiency of the contact tank. It is recommended that the contact tanks should be designed using CFD methods as demonstrated in this study.

\section{Acknowledgements}

The numerical calculations reported in this paper were fully performed at TUBITAK ULAKBIM, High Performance and Grid Computing Center (TRUBA resources).

\section{References}

[1] Çakmakçı, M., Özkaya, B., Yetilmezsoy, K. ve Demir, S. Su Arıtma Tesislerinin Tasarım ve İşletme Esasları, İstanbul, 2013. 
[2] Zhang, J., Martinez, A. E. T. and Zhang, Q., Hydraulic Efficiency in RANS of the Flow in Multi-chambered Contactors, Journal of Hydraulic Engineering, 139, 1150-1157, 2013.

[3] Angeloudis, A., Stoesser, T., Falconer, R.A. and Kim,D.J., Flow, Transport and Disinfection Performance in Small -and Full-Scale Contact Tanks, Journal of HydroEnvironment Research, 9, 15-27, 2015.

[4] Rauen, W.B., Lin, B., Falconer, R.A. and Teixeira, E.C., CFD and Experimental Model Studies for Water Disinfection Tanks with Low Reynolds Number Flows, Chemical Engineering Journal, 137, 550-600, 2008.

[5] Angeloudis, A., Stoesser, T., and Falconer, R.A., Predicting the Disnfection Efficiency Range in Chlorine Contact Tanks Through a CFD-Based Approach, Water Research, 60, 118-129, 2014.

[6] Kim, D., Kim, D.I., Kim, J.H., and Stoesser, T., Large Eddy Simulation of Flow and Tracer Transport in Multichamber Ozone Contactors, Journal of Environmental Engineering, 136, 1, 22-31, 2010.

[7] Wols, B.A., Hofman, J.A.M.H., Uijttewaal, W.S.J., Rietveld, L.C. and Dijk, J.C., Evaluation of Different Disinfection Calculation Methods using CFD, Environmental Modelling \& Software, 25, 573-582, 2010.

[8] Zhang, J., Martinez, A.E.T., Lei, H. and Zhang, Q., Indicators for Technological, Environmental and Economic Sustainablity of Ozone Contactors, Water Research, 101, 606-616, 2016.

[9] Zhang, J., Martinez, A.E.T., and Zhang, Q., Evaluation of Large Eddy Simulation and RANS for Determining Hydraulic Performance of Disinfection Systems for Water Treatment, Journal of Fluids Engineering, 136, 121102-121102-9, 2014.

[10] Kim, D., Elovitz, M., Roberts, P.J.W. and Kim, J.H., Using 3D LIF to Investigate and Improve Performance of a Multichamber Ozone Contactor, Americal Water Works Association Journal, 102, 61-70, 2010.

[11] Zhang, Y., Street, R.L. and Koseff, J.R., A Dynamic Mixed Subgrid-Scale Model and Its Application to Turbulent Recirculating Flows, Physics of Fluids, 5, 3186-3196, 1993.

[12] OpenFOAM, The OpenFOAM Foundation; OpenCFD Ltd.: Bracknell, UK, 2015.

[13] Demirel, E. and Aral, M.M., Unified Analysis of Multi-chamber Contact Tanks and Mixing Efficiency Based on Vorticity Field. Part I: Hydrodynamic Analysis, Water, 8, 495-505, 2016.

[14] U.S. EPA. , Disinfection Profiling and Benchmarking Guidance Manual, Appendix A. Rep. No. EPA-815-R-99-013, 1999, U.S. EPA, Washington, D.C

[15] Demirel, E. and Aral, M.M., Unified Analysis of Multi-Chamber Contact Tanks and Mixing Efficiency Based on Vorticity Field. Part II: Transport Analysis, Water, 8, 537554, 2016. 
An Efficient Contact Tank Design for Potable Water Treatment

[16] Elliot, T., Zeier, B., Xagoraraki, I. and Harrington, G. W., Energy Use at Wisconsin's Drinking Water Facilities, Rep. 2003, 222-1, Energy Center of Wisconsin, Madison, WI.

[17] Aral, M.M. and Demirel, E., Novel Slot-Baffle Design to Improve Mixing Efficiency and Reduce Cost of Disinfection in Drinking Water Treatment, Journal of Environmental Engineering ASCE, 143, 9, 1-5, 2017.

[18] Aral, M.M. and Demirel, E., A New Baffle Design to Improve Mixing and Reduce the Flow Through Energy Requirements in Chlorine and Ozone Contact Tanks, Patent \# 62/498,260, 10 January 2017.

[19] Angeloudis, A., Stoesser, T., Gualtieri, C. and Falconer, R.A., Contact Tanks Design Impact on Process performance, Environ. Model. Assess. 21, 563-576, 2016. 